Commun. Korean Math. Soc. 27 (2012), No. 2, pp. 411-423

http://dx.doi.org/10.4134/CKMS.2012.27.2.411

\title{
OPTIMALITY CONDITIONS AND DUALITY FOR SEMI-INFINITE PROGRAMMING INVOLVING SEMILOCALLY TYPE I-PREINVEX AND RELATED FUNCTIONS
}

\author{
Monika Jaiswal, Shashi Kant Mishra, and Bader Al Shamary
}

\begin{abstract}
A nondifferentiable nonlinear semi-infinite programming problem is considered, where the functions involved are $\eta$-semidifferentiable type I-preinvex and related functions. Necessary and sufficient optimality conditions are obtained for a nondifferentiable nonlinear semi-infinite programming problem. Also, a Mond-Weir type dual and a general MondWeir type dual are formulated for the nondifferentiable semi-infinite programming problem and usual duality results are proved using the concepts of generalized semilocally type I-preinvex and related functions.
\end{abstract}

\section{Introduction}

Generalized convexity plays a central role in mathematical economics and optimization theory. Therefore, generalized convexity is now one of the very active research areas. Various generalizations of convex functions have appeared in literature. Among all generalized convex functions, we use the class of semilocally type I-preinvex functions in this paper.

An important generalization of convex functions termed as semilocally convex functions was introduced by Ewing [2]. Kaul and Kaur [6] defined semilocally quasiconvex and semilocally pseudoconvex functions and obtained sufficient optimality conditions for a class of nonlinear programming problems involving such functions. Kaur [7] obtained necessary optimality conditions and duality results by taking the objective and constraint functions to be semilocally convex and their right differentials at a point to be lower semicontinuous. A significant generalization of convex functions termed preinvex functions was introduced by Weir and Mond [15]. Yang [16] and Li [17] obtained some properties of preinvex function. Noor [10] studied some properties of a class of nonconvex functions, called semipreinvex functions, which includes the classes

Received December 9, 2010.

2010 Mathematics Subject Classification. 90C29, 90C34, 90C46.

Key words and phrases. multiobjective programming, semi-infinite programming, optimality, duality. 
of preinvex functions and arc-connected convex functions. Preda et al. [13] introduced the concepts of semilocally preinvex, semilocally quasi-preinvex and semilocally pseudo-preinvex functions. Fritz John and Kuhn-Tucker necessary optimality conditions and sufficient optimality conditions were given and duality results stated for Wolfe and Mond-Weir types duals using these concepts. Preda and Stancu-Minasian [12] extended these results to a multiple objective programming problem.

In [3], Hanson and Mond introduced two new classes of functions called type I and type II functions, both closely related to, but more general than invex functions. Additional conditions are attached to the Kuhn-Tucker conditions giving a set of conditions which are both necessary and sufficient for optimality in constrained optimization, under appropriate constraint qualifications. Rueda and Hanson [14] defined pseudo-type I and quasi-type I and obtained sufficient optimality criteria for a nonlinear programming problem involving these functions. Hanson et al. [4] extended a (scalarized) generalized type I invexity into a vector invexity (V-type I). A number of sufficiency results and duality theorems have been established under various types of generalized V-type I setting.

Motivated by above discussed papers on generalized type I invexity and the work of Preda [11] and Mishra et al. [8, 9], in this paper, we have obtained necessary and sufficient optimality conditions for a nondifferentiable nonlinear semi-infinite programming problem involving $\eta$-semidifferentiable type Ipreinvex functions. We have also formulated a Mond-Weir dual and a general Mond-Weir dual for a nonlinear semi-infinite programming problem. Duality results are proved using the concepts of generalized semilocally type I-preinvex functions.

\section{Definitions and preliminaties}

In this section we recall, for convenience of reference, a number of basic definitions that will be used throughout the paper.

For $x, y \in \mathbb{R}^{n}$, by $x \leqq y$ we mean $x_{i} \leqq y_{i}$ for all $i, x \leq y$ means $x_{i} \leqq y_{i}$ for all $i$ and $x_{j}<y_{j}$ for at least one $j, 1 \leqq j \leqq n$. By $x<y$ we mean $x_{i}<y_{i}$ for all $i$.

Let $X_{0} \subseteq \mathbb{R}^{n}$ be a set and $\eta: \bar{X}_{0} \times X_{0} \rightarrow \mathbb{R}^{n}$ be a vectorial application. We say that the set $X_{0}$ is $\eta$-vex at $\bar{x} \in X_{0}$ if $\bar{x}+\lambda \eta(x, \bar{x}) \in X_{0}$ for any $x \in X_{0}$ and $\lambda \in[0,1]$. We say that $X_{0}$ is $\eta$-vex if $X_{0}$ is $\eta$-vex at any $x \in X_{0}$.

We remark that if $\eta(x, \bar{x})=x-\bar{x}$ for any $x \in X_{0}$, then $X_{0}$ is $\eta$-vex at $\bar{x}$ if and only if $X_{0}$ is a convex set at $\bar{x}$.

The following definitions are taken from Preda [11].

Definition 1. We say that the set $X_{0} \subseteq \mathbb{R}^{n}$ is an $\eta$-locally starshaped set at $\bar{x}, \bar{x} \in X_{0}$, if for any $x \in X_{0}$, there exists $0<a_{\eta}(x, \bar{x}) \leqq 1$ such that $\bar{x}+\lambda \eta(x, \bar{x}) \in X_{0}$ for any $\lambda \in\left[0, a_{\eta}(x, \bar{x})\right]$.

Definition 2. Let $f: X_{0} \rightarrow \mathbb{R}^{n}$ be a function, where $X_{0} \subseteq \mathbb{R}^{n}$ is an $\eta$-locally starshaped set at $\bar{x} \in X_{0}$. We say that $f$ is: 
(i) semilocally preinvex (slpi) at $\bar{x}$ if, corresponding to $\bar{x}$ and each $x \in X_{0}$, there exists a positive number $d_{\eta}(x, \bar{x}) \leqq a_{\eta}(x, \bar{x})$ such that $f(\bar{x}+\lambda \eta(x, \bar{x}))$ $\leqq \lambda f(x)+(1-\lambda) f(\bar{x})$ for $0<\lambda<d_{\eta}(x, \bar{x})$;

(ii) semilocally quasi-preinvex (slqpi) at $\bar{x}$ if, corresponding to $\bar{x}$ and each $x \in$ $X_{0}$, there exists a positive number $d_{\eta}(x, \bar{x}) \leqq a_{\eta}(x, \bar{x})$ such that $f(x) \leqq f(\bar{x})$ and $0<\lambda<d_{\eta}(x, \bar{x})$ implies $f(\bar{x}+\lambda \eta(x, \bar{x})) \leqq f(\bar{x})$.

Definition 3. Let $f: X_{0} \rightarrow \mathbb{R}^{n}$ be a function, where $X_{0} \subseteq \mathbb{R}^{n}$ is an $\eta$ locally starshaped set at $\bar{x} \in X_{0}$. We say that $f$ is $\eta$-semidifferentiable at $\bar{x}$ if $(d f)^{+}(\bar{x}, \eta(x, \bar{x}))$ exists for each $x \in X_{0}$, where

$$
(d f)^{+}(\bar{x}, \eta(x, \bar{x}))=\lim _{\lambda \rightarrow 0^{+}} \frac{1}{\lambda}[f(\bar{x}+\lambda \eta(x, \bar{x}))-f(\bar{x})]
$$

(the right derivative at $\bar{x}$ along the direction $\eta(x, \bar{x})$ ).

If $f$ is $\eta$-semidifferentiable at any $\bar{x} \in X_{0}$, then $f$ is said to be $\eta$-semidifferentiable on $X_{0}$.

Definition 4. We say that $f$ is semilocally pseudo-preinvex (slppi) at $\bar{x}$ if for any $x \in X_{0}$,

$$
(d f)^{+}(\bar{x}, \eta(x, \bar{x})) \geqq 0 \Rightarrow f(x) \geqq f(\bar{x}) .
$$

If $f$ slppi at any $\bar{x} \in X_{0}$, then $f$ is said to be slppi on $X_{0}$.

Theorem 1. Let $f: X_{0} \rightarrow \mathbb{R}^{n}$ be an $\eta$-semidifferentiable function at $\bar{x} \in X_{0}$. If $f$ is slqpi at $\bar{x}$ and $f(x) \leqq f(\bar{x})$, then $(d f)^{+}(\bar{x}, \eta(x, \bar{x})) \leqq 0$.

Definition 5 (Elster and Nehse [1]). A function $f: X_{0} \rightarrow \mathbb{R}^{n}$ is a convexlike function if for any $x, y \in X_{0}$ and $0 \leqq \lambda \leqq 1$, there is $z \in X_{0}$ such that

$$
f(z) \leqq \lambda f(x)+(1-\lambda) f(y) .
$$

Remark 1. The convex and the preinvex functions are convexlike.

Lemma 1 (Hayashi and Komiya [5]). Let $S$ be a nonempty set in $\mathbb{R}^{n}$ and $\psi: S \rightarrow \mathbb{R}^{k}$ be a convexlike function. Then either

$$
\psi(x)<0 \text { has a solution } x \in S
$$

or

$$
\lambda^{T} \psi(x) \geqq 0 \text { for all } x \in S,
$$

for some $\lambda \in \mathbb{R}^{k}, \lambda \geq 0$, but both alternatives are never true.

Theorem 2. Let $\bar{x} \in X$ be a (local) weak minimum solution for the following problem:

$$
\begin{gathered}
\text { minimize }\left(f_{1}(x), \ldots, f_{p}(x)\right) \\
\text { subject to } g\left(x, u^{i}\right) \leqq 0, i \in I, \\
x \in X_{0},
\end{gathered}
$$


where $U \subset \mathbb{R}^{m}$ is an infinite countable set, $f=\left(f_{1}, \ldots, f_{p}\right): X_{0} \rightarrow \mathbb{R}^{p}$ and $g: X_{0} \times U \rightarrow \mathbb{R}^{m}$ are $\eta$-semidifferentiable at $\bar{x}$. Also, we assume that $g\left(x, u^{i}\right)(i \in J(\bar{x}))$ is a continuous function at $\bar{x}$ and $(d f)^{+}(\bar{x}, \eta(x, \bar{x}))$ and $(d g)^{+}\left((\bar{x}, \eta(x, \bar{x})), u^{i}\right)$ are convexlike functions on $X_{0}$, where $I=\left\{i: g\left(x, u^{i}\right)\right.$ $\left.\leqq 0, x \in X_{0}, u^{i} \in U\right\}$, the set of $i$ for which our constraint is active, $I(\bar{x})=$ $\left.\overline{\{i}: g\left(x, u^{i}\right)=0, x \in X_{0}, u^{i} \in U\right\}$ and $J(\bar{x})=\left\{i: g\left(x, u^{i}\right)<0, x \in X_{0}, u^{i} \in U\right\}$. If $g\left(x, u^{i}\right)$ satisfies a regularity condition at $\bar{x}$ (see [12, Definition 3.2]), then there exist $\lambda^{0} \in \mathbb{R}^{p}$ and $\lambda=\left(\lambda_{i}\right)_{i \in I} \in \mathbb{R}^{m}$ such that

$$
\begin{gathered}
\lambda^{0^{T}}(d f)^{+}(\bar{x}, \eta(x, \bar{x}))+\sum_{i \in I} \lambda_{i}(d g)^{+}\left((\bar{x}, \eta(x, \bar{x})), u^{i}\right) \text { for all } x \in X_{0}, \\
\sum_{i \in I} \lambda_{i} g\left(\bar{x}, u^{i}\right)=0, g\left(\bar{x}, u^{i}\right) \leqq 0, \\
\lambda^{0^{T}} e=1, \lambda^{0} \geq 0, \lambda \geqq 0,
\end{gathered}
$$

where $e=(1, \ldots, 1)^{T} \in \mathbb{R}^{p}$.

\section{Necessary optimality conditions}

In this paper, we consider the following nonlinear semi-infinite programming problem $(\mathrm{P})$ :

$$
\begin{gathered}
\operatorname{minimize}\left(f_{1}(x), \ldots, f_{p}(x)\right) \\
\text { subject to } g(x, u) \leqq 0, \quad(u \in U) \\
x \in X_{0},
\end{gathered}
$$

where $X_{0} \subseteq \mathbb{R}^{n}$ is a nonempty set and $U \subset \mathbb{R}^{m}$ is an infinite countable set. Let $f=\left(f_{1}, \ldots, f_{p}\right): X_{0} \rightarrow \mathbb{R}^{p}, g: X_{0} \times U \rightarrow \mathbb{R}^{m}$. We put $X=$ $\left\{x \in X_{0}: g\left(x, u^{i}\right) \leqq 0, x \in u^{i} \in U\right\}$ for the feasible set of problem (P).

The following definitions are taken from Mishra et al. [8].

Definition 6. We say that the problem $(\mathrm{P})$ is $\eta$-semidifferentiable type Ipreinvex at $\bar{x}$ if for any $x \in X_{0}$, we have

$$
\begin{gathered}
f_{j}(x)-f_{j}(\bar{x}) \geqq\left(d f_{j}\right)^{+}(\bar{x}, \eta(x, \bar{x})), \forall j \in M=\{1,2, \ldots, p\}, \\
-g\left(\bar{x}, u^{i}\right) \geqq(d g)^{+}\left((\bar{x}, \eta(x, \bar{x})), u^{i}\right), \forall i \in I .
\end{gathered}
$$

Definition 7. (i) We say that the problem (P) is $\eta$-semidifferentiable pseudoquasi-type I-preinvex at $\bar{x}$ if for any $x \in X_{0}$, we have

$$
\begin{gathered}
\left(d f_{j}\right)^{+}(\bar{x}, \eta(x, \bar{x})) \geqq 0 \Rightarrow f_{j}(x) \geqq f_{j}(\bar{x}), \forall j \in M, \\
-g\left(\bar{x}, u^{i}\right) \leqq 0 \Rightarrow(d g)^{+}\left((\bar{x}, \eta(x, \bar{x})), u^{i}\right) \leqq 0, \forall i \in I .
\end{gathered}
$$

The problem $(\mathrm{P})$ is $\eta$-semidifferentiable pseudo-quasi-type I-preinvex on $X_{0}$ if it is $\eta$-semidifferentiable pseudo-quasi-type I-preinvex at any $\bar{x} \in X_{0}$.

(ii) We say that the problem (P) is $\eta$-semidifferentiable strict pseudo-quasitype I-preinvex at $\bar{x}$ if for any $x \in X_{0}$ and $x \neq \bar{x}$, we have

$$
\left(d f_{j}\right)^{+}(\bar{x}, \eta(x, \bar{x})) \geqq 0 \Rightarrow f_{j}(x)>f_{j}(\bar{x}), \forall j \in M,
$$




$$
-g\left(\bar{x}, u^{i}\right) \leqq 0 \Rightarrow(d g)^{+}\left((\bar{x}, \eta(x, \bar{x})), u^{i}\right) \leqq 0, \forall i \in I .
$$

The problem (P) is $\eta$-semidifferentiable strict pseudo-quasi-type I-preinvex on $X_{0}$ if it is $\eta$-semidifferentiable strict pseudo-quasi-type I-preinvex at any $\bar{x} \in X_{0}$.

Definition 8. We say that the problem $(\mathrm{P})$ is $\eta$-semidifferentiable quasipseudo-type I-preinvex at $\bar{x}$ if for any $x \in X_{0}$, we have

$$
\begin{gathered}
f_{j}(x) \leqq f_{j}(\bar{x}) \Rightarrow\left(d f_{j}\right)^{+}(\bar{x}, \eta(x, \bar{x})) \leqq 0, \forall j \in M, \\
(d g)^{+}\left((\bar{x}, \eta(x, \bar{x})), u^{i}\right) \geqq 0 \Rightarrow-g\left(\bar{x}, u^{i}\right) \geqq 0, \quad \forall i \in I .
\end{gathered}
$$

The problem $(\mathrm{P})$ is $\eta$-semidifferentiable quasi-pseudo-type I-preinvex on $X_{0}$ if it is $\eta$-semidifferentiable quasi-pseudo-type I-preinvex at any $\bar{x} \in X_{0}$.

Definition 9. For the problem (P), a point $\bar{x} \in X$ is said to be a weak minimum if there exists no feasible point $x$ for which $f(\bar{x})>f(x)$.

For $\bar{x} \in X$, we put $I(\bar{x})=\left\{i: g\left(x, u^{i}\right)=0, x \in X_{0}, u^{i} \in U\right\}, g^{0}=g\left(x, u^{i}\right)$, $i \in I(\bar{x})$ and $J(\bar{x})=\left\{i: g\left(x, u^{i}\right)<0, x \in X_{0}, u^{i} \in U\right\}$.

Definition 10. We say that $(\mathrm{P})$ satisfies the generalized Slater's constraint qualification (GSCQ) at $\bar{x} \in X$ if $g^{0}$ is slppi at $\bar{x}$ and there exists an $\hat{x} \in X_{0}$ such that $g^{0}\left(\hat{x}, u^{i}\right)<0$.

Lemma 2. Let $\bar{x} \in X$ be a (local) weak minimum solution for $(\mathrm{P})$. Further, we assume that $g\left(x, u^{i}\right)$ is continuous at $\bar{x}$ for any $i \in J(\bar{x})$ and $f, g^{0}$ are $\eta$-semidifferentiable at $\bar{x}$. Then the system

$$
(d f)^{+}(\bar{x}, \eta(x, \bar{x}))<0,
$$

has no solution $x \in X_{0}$.

Proof. Let $\bar{x}$ be a (local) weak minimum solution for (P) and suppose there exists $x^{*} \in X$ such that

$$
\begin{gathered}
(d f)^{+}\left(\bar{x}, \eta\left(x^{*}, \bar{x}\right)\right)<0, \\
\left(d g^{0}\right)^{+}\left(\left(\bar{x}, \eta\left(x^{*}, \bar{x}\right)\right), u^{i}\right)<0 .
\end{gathered}
$$

Let $\varphi\left(\bar{x}, x^{*}, \lambda\right)=f\left(\bar{x}+\lambda \eta\left(x^{*}, \bar{x}\right)\right)-f(\bar{x})$. We have $\varphi\left(\bar{x}, x^{*}, 0\right)=0$ and the right differential of $\varphi\left(\bar{x}, x^{*}, 0\right)$ with respect to $\lambda$ at $\lambda=0$ is given by

$$
\lim _{\lambda \rightarrow 0} \frac{1}{\lambda}\left[\varphi\left(\bar{x}, x^{*}, \lambda\right)-\varphi\left(\bar{x}, x^{*}, 0\right)\right]=\lim _{\lambda \rightarrow 0} \frac{1}{\lambda}\left[f\left(\bar{x}+\lambda \eta\left(x^{*}, \bar{x}\right)\right)-f(\bar{x})\right]
$$

or

$$
\lim _{\lambda \rightarrow 0} \frac{1}{\lambda}\left[\varphi\left(\bar{x}, x^{*}, \lambda\right)-\varphi\left(\bar{x}, x^{*}, 0\right)\right]=(d f)^{+}\left(\bar{x}, \eta\left(x^{*}, \bar{x}\right)\right) .
$$

Using (2), we get

$$
\lim _{\lambda \rightarrow 0} \frac{1}{\lambda}\left[\varphi\left(\bar{x}, x^{*}, \lambda\right)-\varphi\left(\bar{x}, x^{*}, 0\right)\right]<0 .
$$


Hence, $\varphi\left(\bar{x}, x^{*}, \lambda\right)<0$ if $\lambda$ is in some open interval $\left(0, \delta_{1}\right), \delta_{1}>0$; i.e.,

$$
f\left(\bar{x}+\lambda \eta\left(x^{*}, \bar{x}\right)\right)<f(\bar{x}), \lambda \in\left(0, \delta_{1}\right) .
$$

Similarly, we get

$$
g^{0}\left(\left(\bar{x}+\lambda \eta\left(x^{*}, \bar{x}\right)\right), u^{i}\right)<g^{0}\left(\bar{x}, u^{i}\right)=0, \lambda \in\left(0, \delta_{2}\right), \delta_{2}>0 .
$$

Now, for $i \in J(\bar{x}), g\left(x, u^{i}\right)<0$ and $g\left(x, u^{i}\right)$ is continuous at $\bar{x}$, therefore, there exists $\delta^{\prime}>0$ such that

$$
g\left(\left(\bar{x}+\lambda \eta\left(x^{*}, \bar{x}\right)\right), u^{i}\right)<0, \lambda \in\left(0, \delta^{\prime}\right) \text { for any } i \in J(\bar{x}) .
$$

Let $\delta=\min \left(\delta_{1}, \delta_{2}, \delta^{\prime}\right)$. Then

$$
\bar{x}+\lambda \eta\left(x^{*}, \bar{x}\right) \in S_{\delta}(\bar{x}) \subseteq N_{\delta}(\bar{x}), \lambda \in(0, \delta),
$$

where $S_{\delta}(\bar{x})$ is a hypersphere around $\bar{x}$ and $N_{\delta}(\bar{x})$ is a neighbourhood of $\bar{x}$. Now, we have

$$
\begin{gathered}
f\left(\bar{x}+\lambda \eta\left(x^{*}, \bar{x}\right)\right)<f(\bar{x}), \\
g\left(\left(\bar{x}+\lambda \eta\left(x^{*}, \bar{x}\right)\right), u^{i}\right)<0
\end{gathered}
$$

for any $\lambda \in(0, \delta)$.

By (4) and (6), we have $\bar{x}+\lambda \eta\left(x^{*}, \bar{x}\right) \in X_{0} \cap N_{\delta}(\bar{x})$ for any $\lambda \in(0, \delta)$.

Using (5), for $Q(x)=\left(f_{1}(x), \ldots, f_{p}(x)\right)$, we get

$$
Q\left(\bar{x}+\lambda \eta\left(x^{*}, \bar{x}\right)\right)<Q(\bar{x}),
$$

which contradicts the assumption that $\bar{x}$ is a (local) weak minimum solution of (P). Hence there exists no $x \in X_{0}$ satisfying the system (1). Thus the lemma is proved.

In the next theorem, we obtain an important result of Fritz-John type necessary optimality criteria.

Theorem 3 (Fritz-John type necessary optimality criteria). Let us suppose that $g\left(x, u^{i}\right)$ is continuous at $\bar{x}$ for $i \in J(\bar{x}),(d f)^{+}(\bar{x}, \eta(x, \bar{x}))$ and $\left(d g^{0}\right)^{+}((\bar{x}$, $\left.\eta(x, \bar{x})), u^{i}\right)$ are convexlike functions on $X_{0}$. If $\bar{x}$ is a (local) weak minimum solution for $(\mathrm{P})$, then there exist $\lambda^{0} \in \mathbb{R}^{p}$ and $\lambda=\left(\lambda_{i}\right)_{i \in I} \in \mathbb{R}^{m}$ such that

$$
\sum_{i \in I} \lambda_{i} g\left(\bar{x}, u^{i}\right)=0
$$

$$
\left(\lambda^{0}, \lambda\right) \neq 0, \quad\left(\lambda^{0}, \lambda\right) \geqq 0 .
$$


Proof. If $\bar{x}$ is a (local) weak minimum solution for $(\mathrm{P})$ then, by Lemma 2 , the system (1) has no solution $x \in X_{0}$. But the assumption of Lemma 1 also holds and since the system (1) has no solution $x \in X_{0}$, we obtain that there exist $\lambda^{0} \in$ $\mathbb{R}^{p}, \lambda_{i} \in \mathbb{R}(i \in I(\bar{x}))$, such that $\lambda^{0} \geqq 0, \lambda_{i} \geqq 0(i \in I(\bar{x})),\left(\lambda^{0},\left(\lambda_{i}\right)_{i \in I(\bar{x})}\right) \neq 0$, with

(10)

$$
\lambda^{0^{T}}(d f)^{+}(\bar{x}, \eta(x, \bar{x}))+\sum_{i \in I(\bar{x})} \lambda_{i}\left(d g^{0}\right)^{+}\left((\bar{x}, \eta(x, \bar{x})), u^{i}\right) \geqq 0 \text { for all } x \in X_{0} .
$$

If we put $\lambda_{i}=0$ for $i \in J(\bar{x})$, by (10) we get (7). Finally, the relation (6) follows obviously and the proof is complete.

Theorem 4 (Karush-Kuhn-Tucker type necessary optimality criterion). Let $\bar{x} \in X$ be a (local) weak minimum solution for $(\mathrm{P})$, let $g\left(x, u^{i}\right)$ be continuous at $\bar{x}$ for $i \in J(\bar{x})$ and let $\left(d f_{j}\right)^{+}(\bar{x}, \eta(x, \bar{x}))(j=1,2, \ldots, p)$ and $\left(d g^{0}\right)^{+}((\bar{x}$, $\left.\eta(x, \bar{x})), u^{i}\right)$ be convexlike functions on $X_{0}$. If $g$ satisfies (GSQ) at $\bar{x}$, then there exist $\lambda^{0} \in \mathbb{R}_{+}^{p}, \lambda \in \mathbb{R}^{m}$, such that

$$
\begin{gathered}
\sum_{j=1}^{p} \lambda_{j}^{0}\left(d f_{j}\right)^{+}(\bar{x}, \eta(x, \bar{x}))+\sum_{i \in I} \lambda_{i}(d g)^{+}\left((\bar{x}, \eta(x, \bar{x})), u^{i}\right) \geqq 0 \text { for all } x \in X_{0}, \\
\qquad \sum_{i \in I} \lambda_{i} g\left(\bar{x}, u^{i}\right)=0, g\left(\bar{x}, u^{i}\right) \leqq 0, \\
\qquad \lambda^{0^{T}} e=1, \quad \lambda^{0} \geq 0, \lambda \geqq 0,
\end{gathered}
$$

Proof. If $\bar{x}$ is a (local) weak minimum solution for (P). Now, applying Theorem 2 to problem $(\mathrm{P})$, we get that there exist, $\lambda^{0} \in \mathbb{R}_{+}^{p}, \lambda \in \mathbb{R}^{m}$ such that

$$
\begin{gathered}
\sum_{j=1}^{p} \lambda_{j}^{0}\left(d f_{j}\right)^{+}(\bar{x}, \eta(x, \bar{x}))+\sum_{i \in I} \lambda_{i}(d g)^{+}\left((\bar{x}, \eta(x, \bar{x})), u^{i}\right) \geqq 0 \text { for all } x \in X_{0}, \\
\sum_{i \in I} \lambda_{i} g\left(\bar{x}, u^{i}\right)=0, \\
g\left(\bar{x}, u^{i}\right) \leqq 0, \\
(12) \quad \lambda^{0^{T}} e=1, \\
\lambda^{0} \geq 0, \quad \lambda \geqq 0,
\end{gathered}
$$

and the theorem is proved. 


\section{Sufficient optimality criteria}

In this section, using the concept of (locally) weak optimality, we give some sufficient optimality conditions for the problem $(\mathrm{P})$.

Theorem 5. Let $\bar{x} \in X$ and $(\mathrm{P})$ be $\eta$-semilocally type I-preinvex at $\bar{x}$. Also, we assume that there exist $\lambda^{0} \in \mathbb{R}^{p}$ and $\lambda \in \mathbb{R}^{m}$ such that

(16)

$$
\sum_{j=1}^{p} \lambda_{j}^{0}\left(d f_{j}\right)^{+}(\bar{x}, \eta(x, \bar{x}))+\sum_{i \in I} \lambda_{i}(d g)^{+}\left((\bar{x}, \eta(x, \bar{x})), u^{i}\right) \geqq 0 \text { for all } x \in X,
$$

$$
\begin{gathered}
\sum_{i \in I} \lambda_{i} g\left(\bar{x}, u^{i}\right)=0, \\
\lambda^{0^{T}} e=1, \\
\lambda^{0} \geq 0, \quad \lambda \geqq 0 .
\end{gathered}
$$

Then $\bar{x}$ is a weak minimum solution for $(\mathrm{P})$.

Proof. We proceed by contradiction. Let there exists $\tilde{x} \in X$ such that

$$
f_{j}(\tilde{x})<f_{j}(\bar{x}) \text { for any } j \in M \text {, where } M=\{1,2, \ldots, p\} \text {. }
$$

Since $(\mathrm{P})$ is $\eta$-semilocally type I-preinvex at $\bar{x}$, we get

$$
f_{j}(\tilde{x})-f_{j}(\bar{x}) \geqq\left(d f_{j}\right)^{+}(\bar{x}, \eta(\tilde{x}, \bar{x})), j \in M,
$$

and

$$
-g\left(\bar{x}, u^{i}\right) \geqq(d g)^{+}\left((\bar{x}, \eta(\tilde{x}, \bar{x})), u^{i}\right), i \in I .
$$

Multiplying (21) by $\lambda_{j}^{0} \geqq 0, j \in M, \lambda^{0} \in \mathbb{R}_{p}$, (22) by $\lambda_{i} \geqq 0, i \in I$, and then summing the obtained relations, we get

$$
\begin{aligned}
& \sum_{j=1}^{p} \lambda_{j}^{0}\left(f_{j}(\tilde{x})-f_{j}(\bar{x})\right)-\sum_{i \in I} \lambda_{i} g\left(\bar{x}, u^{i}\right) \\
\geqq & \sum_{j=1}^{p} \lambda_{j}^{0}\left(d f_{j}\right)^{+}(\bar{x}, \eta(\tilde{x}, \bar{x}))+\sum_{i \in I} \lambda_{i}(d g)^{+}\left((\bar{x}, \eta(\tilde{x}, \bar{x})), u^{i}\right) \geqq 0,
\end{aligned}
$$

where the last inequality is according to (16). Hence,

$$
\sum_{j=1}^{p} \lambda_{j}^{0}\left(f_{j}(\tilde{x})-f_{j}(\bar{x})\right)-\sum_{i \in I} \lambda_{i} g\left(\bar{x}, u^{i}\right) \geqq 0 .
$$

Since $x \in X, \lambda \geqq 0$, by (17) and (23), we get

$$
\sum_{j=1}^{p} \lambda_{j}^{0}\left(f_{j}(\tilde{x})-f_{j}(\bar{x})\right) \geqq 0 .
$$


Using (18), (19) and (24), we obtain that there exists $j_{0} \in M$ such that

$$
f_{j_{0}}(\tilde{x}) \geqq f_{j_{0}}(\bar{x}),
$$

which is a contradiction to (20). Thus, the theorem is proved and $\bar{x}$ is a weak minimum solution for $(\mathrm{P})$.

Theorem 6. Let $\bar{x} \in X, \lambda^{0} \in \mathbb{R}^{p}$ and $\lambda \in \mathbb{R}^{m}$ such that the conditions (16)-(19) of Theorem 5 hold. Moreover, we assume that $(\mathrm{P})$ is $\eta$-semilocally pseudo-quasi-type I-preinvex at $\bar{x}$. Then $\bar{x}$ is a weak minimum solution for $(\mathrm{P})$.

Proof. We assume that $\bar{x}$ is not a weak minimum solution for $(\mathrm{P})$. Then there exists $\tilde{x} \in X$ such that

$$
f_{j}(\tilde{x})<f_{j}(\bar{x}) \text { for any } j \in M \text {. }
$$

Now, by the $\eta$-semilocally pseudo-quasi-type I-preinvexity of $(\mathrm{P})$ at $\bar{x}$, we get

$$
\left(d f_{j}\right)^{+}(\bar{x}, \eta(\tilde{x}, \bar{x}))<0 \text { for any } j \in M
$$

Using $\lambda_{j}^{0} \in \mathbb{R}_{+}^{p}, \lambda^{0^{T}} e=1$, we obtain

$$
\sum_{j=1}^{p} \lambda_{j}^{0}\left(d f_{j}\right)^{+}(\bar{x}, \eta(\tilde{x}, \bar{x}))<0
$$

For $i \in I(\bar{x}), g\left(\bar{x}, u^{i}\right)=0$.

Hence,

$$
-g\left(\bar{x}, u^{i}\right) \leqq 0 \text { for any } i \in I(\bar{x})
$$

Now, again by $\eta$-semilocally pseudo-quasi-type I-preinvexity of $(\mathrm{P})$ at $\bar{x}$, we obtain

$$
(d g)^{+}\left((\bar{x}, \eta(\tilde{x}, \bar{x})), u^{i}\right) \leqq 0 \text { for any } i \in I(\bar{x}) .
$$

But $\lambda \in \mathbb{R}_{+}^{m}$ and then taking $\lambda_{i}=0$ for $i \in J(\bar{x})$, we get

$$
\sum_{i \in I} \lambda_{i}(d g)^{+}\left((\bar{x}, \eta(\tilde{x}, \bar{x})), u^{i}\right) \leqq 0 .
$$

Now, by (25) and (26), we obtain

$$
\sum_{j=1}^{p} \lambda_{j}^{0}\left(d f_{j}\right)^{+}(\bar{x}, \eta(\tilde{x}, \bar{x}))+\sum_{i \in I} \lambda_{i}(d g)^{+}\left((\bar{x}, \eta(\tilde{x}, \bar{x})), u^{i}\right)<0,
$$

which is a contradiction to (16). Hence is a weak minimum for $(\mathrm{P})$ and the theorem is proved. 


\section{Duality}

We consider, for (P), a Mond-Weir dual (MWD) as maximize $f(y)$

subject to

(27)

$$
\sum_{j=1}^{p} \lambda_{j}^{0}\left(d f_{j}\right)^{+}(y, \eta(x, y))+\sum_{i \in I} \lambda_{i}(d g)^{+}\left((y, \eta(x, y)), u^{i}\right) \geqq 0 \text { for all } x \in X,
$$

$$
\begin{gathered}
\sum_{i \in I} \lambda_{i} g\left(y, u^{i}\right) \geqq 0, \\
\lambda^{0^{T}} e=1, \quad \lambda^{0} \geq 0, \quad \lambda^{0} \in \mathbb{R}^{p},
\end{gathered}
$$

$$
\lambda \geqq 0, \quad y \in X_{0} .
$$

Let $W$ denote the set of all feasible solutions of (MWD). Now we establish certain duality results between (P) and (MWD). Assume that $f$ and $g$ are $\eta$-semidifferentiable on $X$.

Theorem 7 (Weak duality). Assume that $x$ is feasible for $(\mathrm{P})$ and $\left(y, u^{i}, \lambda^{0}, \lambda\right)$ is feasible for (WMD). If the pair of functions $f_{j}($.$) and \sum_{i \in I} \lambda_{i} g\left(., u^{i}\right)$ is $\eta$ semilocally pseudo-quasi-type I-preinvex at $y$ for all $j \in M$, then

$$
f_{j}(y) \leqq f_{j}(x) \text { for any } j \in M \text {. }
$$

Proof. Suppose on contrary that

$$
f_{j}(y)>f_{j}(x) \text { for any } j \in M .
$$

Since the pair of functions $f_{j}(\cdot)$ and $\sum_{i \in I} \lambda_{i} g\left(\cdot, u^{i}\right)$ is $\eta$-semilocally pseudoquasi-type I-preinvex at $y$ for all $j \in M$, from (30) we get

$$
\left(d f_{j}\right)^{+}(y, \eta(x, y))<0 \text { for any } j \in M .
$$

Using $\lambda_{j}^{0} \in \mathbb{R}_{+}^{p}, \lambda^{0^{T}} e=1$, we obtain

$$
\sum_{j=1}^{p} \lambda_{j}^{0}\left(d f_{j}\right)^{+}(y, \eta(x, y))<0 .
$$

Now, from (28), we get

$$
-\sum_{i \in I} \lambda_{i} g\left(y, u^{i}\right) \leqq 0
$$

Hence, we have

$$
\sum_{i \in I} \lambda_{i}(d g)^{+}\left((y, \eta(x, y)), u^{i}\right) \leqq 0 .
$$


Adding (31) and (32), we get

$$
\sum_{j=1}^{p} \lambda_{j}^{0}\left(d f_{j}\right)^{+}(y, \eta(x, y))+\sum_{i \in I} \lambda_{i}(d g)^{+}\left((y, \eta(x, y)), u^{i}\right)<0,
$$

which is a contradiction to (27). Hence,

$$
f_{j}(y) \leqq f_{j}(x) \text { for any } j \in M \text {. }
$$

Now, we consider, for (P), a general Mond-Weir dual (GMWD) as

subject to

$$
\text { maximize } f+\sum_{i \in I_{0}} \lambda_{i} g\left(y, u^{i}\right)
$$

$$
\sum_{j=1}^{p} \lambda_{j}^{0}\left(d f_{j}\right)^{+}(y, \eta(x, y))+\sum_{i \in I} \lambda_{i}(d g)^{+}\left((y, \eta(x, y)), u^{i}\right) \geqq 0 \text { for all } x \in X
$$

$$
\begin{aligned}
& \sum_{i \in I_{s}} \lambda_{i} g\left(y, u^{i}\right) \geqq 0 \quad(1 \leqq s \leqq \gamma), \\
& \lambda^{0^{T}} e=1, \quad \lambda^{0} \geq 0, \quad \lambda^{0} \in \mathbb{R}^{p},
\end{aligned}
$$

$$
\lambda \geqq 0, \quad y \in X_{0},
$$

where $\gamma \geqq 1, I_{s} \cap I_{t}=\phi$ for $s \neq t$ and $\bigcup_{s=0}^{\gamma} I_{s}=I$.

Let $\overline{W^{\prime}}$ denote the set of all feasible solutions of (GMWD). Also, we define the following sets:

$$
A=\left\{\left(\lambda^{0}, \lambda\right) \in \mathbb{R}^{p} \times \mathbb{R}^{m}:\left(y, u^{i}, \lambda^{0}, \lambda\right) \in W^{\prime} \text { for some } y \in X_{0}\right\},
$$

and, for $\left(\lambda^{0}, \lambda\right) \in A$,

$$
B\left(\lambda^{0}, \lambda\right)=\left\{y \in X_{0}:\left(y, u^{i}, \lambda^{0}, \lambda\right) \in W^{\prime}\right\} .
$$

We put $B=\bigcup_{\left(\lambda^{0}, \lambda\right) \in A} B\left(\lambda^{0}, \lambda\right)$ and note that $B \subset X_{0}$. Also, we note that if $\left(y, u^{i}, \lambda^{0}, \lambda\right) \in W^{\prime}$, then $\left(\lambda^{0}, \lambda\right) \in A$ and $y \in B\left(\lambda^{0}, \lambda\right)$.

Now we establish certain duality results between (P) and (GMWD). Assume that $f$ and $g$ are $\eta$-semidifferentiable on $X$.

Theorem 8 (Weak duality). Assume that for all feasible solutions $x \in X$ and $\left(y, u^{i}, \lambda^{0}, \lambda\right) \in W^{\prime}$ for $(\mathrm{P})$ and (GMWD), respectively, the pair of functions $f_{j}(\cdot)+\sum_{i \in I_{0}} \lambda_{i} g\left(\cdot, u^{i}\right)$ and $\sum_{i \in I_{s}} \lambda_{i}^{0} g\left(y, u^{i}\right)$, for $1 \leqq s \leqq \gamma$, is $\eta$-semilocally strict pseudo-quasi-type I-preinvex at $y$ on $B\left(\lambda^{0}, \lambda\right)$ for all $j \in M$. Then the following cannot hold:

$$
f_{j}(x) \leqq f_{j}(y)+\sum_{i \in I_{0}} \lambda_{i} g\left(y, u^{i}\right) \text { for any } j \in M .
$$


Proof. Using the feasibility of $\left(y, u^{i}, \lambda^{0}, \lambda\right)$ for (GMWD), we obtain

$$
-\sum_{i \in I_{s}} \lambda_{i} g\left(y, u^{i}\right) \leqq 0 \text { for all } s, 1 \leqq s \leqq \gamma
$$

By (37) and assumption of the theorem, we get

$$
\sum_{i \in I_{s}} \lambda_{i}(d g)^{+}\left((y, \eta(x, y)), u^{i}\right) \leqq 0, \quad 1 \leqq s \leqq \gamma .
$$

Now, we suppose to the contrary that (36) hold. Hence, using (36) and feasibility of $x$ for $(\mathrm{P})$, we obtain

$$
f_{j}(x)+\sum_{i \in I_{0}} \lambda_{i} g\left(x, u^{i}\right) \leqq f_{j}(y)+\sum_{i \in I_{0}} \lambda_{i} g\left(y, u^{i}\right) \text { for any } j \in M .
$$

Using assumption of the theorem and (39), we can have

(40) $\left(d f_{j}\right)^{+}(y, \eta(x, y))+\sum_{i \in I_{0}} \lambda_{i}(d g)^{+}\left((y, \eta(x, y)), u^{i}\right)<0$ for any $j \in M$.

By (35) and (40), we get

$$
\sum_{j=1}^{p} \lambda_{j}^{0}\left(d f_{j}\right)^{+}(y, \eta(x, y))+\sum_{i \in I_{0}} \lambda_{i}(d g)^{+}\left((y, \eta(x, y)), u^{i}\right)<0 .
$$

Now, by (33) and above equation, we obtain

$$
\sum_{s=1}^{\gamma} \sum_{i \in I_{s}} \lambda_{i}(d g)^{+}\left((y, \eta(x, y)), u^{i}\right)>0,
$$

which is a contradiction to (38). Thus, the theorem is proved.

Theorem 9 (Weak duality). Assume that for all feasible solutions $x \in X$ and $\left(y, u^{i}, \lambda^{0}, \lambda\right) \in W^{\prime}$ for $(\mathrm{P})$ and (GMWD), respectively, the pair of functions $f_{j}(\cdot)+\sum_{i \in I_{0}} \lambda_{i} g\left(\cdot, u^{i}\right)$ and $\sum_{i \in I_{s}} \lambda_{i}^{0} g\left(y, u^{i}\right)$, for $1 \leqq s \leqq \gamma$, is $\eta$-semilocally pseudo-quasi-type I-preinvex at $y$ on $B\left(\lambda^{0}, \lambda\right)$ for all $j \in M$ with $\lambda_{0}>0$. Then the following cannot hold:

$$
f_{j}(x) \leqq f_{j}(y)+\sum_{i \in I_{0}} \lambda_{i} g\left(y, u^{i}\right) \text { for any } j \in M .
$$

Proof. The proof is very similar to the proof of Theorem 8 .

\section{References}

[1] K. H. Elster and R. Nehse, Optimality Conditions for some Nonconvex Problems, Springer-Verlag, New York, 1980.

[2] G. M. Ewing, Sufficient conditions for global minima of suitably convex functionals from variational and control theory, SIAM Rev. 19 (1977), no. 2, 202-220.

[3] M. A. Hanson and B. Mond, Necessary and sufficient conditions in constrained optimization, Report M683, Department of Statistics, Florida State University, Tallahassee, Florida, 1984.

[4] M. A. Hanson, R. Pini, and C. Singh, Multiobjective programming under generalized type I invexity, J. Math. Anal. Appl. 261 (2001), no. 2, 562-577. 
[5] M. Hayashi and H. Komiya, Perfect duality for convexlike programs, J. Optim. Theory Appl. 38 (1982), no. 2, 179-189.

[6] R. N. Kaul and S. Kaur, Generalizations of convex and related functions, European J. Oper. Res. 9 (1982), no. 4, 369-377.

[7] S. Kaur, Theoretical studies in mathematical programming, Ph.D. Thesis, University of Delhi, India, 1984

[8] S. K. Mishra, S. Y. Wang, and K. K. Lai, Multiple objective fractional programming involving semilocally type I-preinvex and related functions, J. Math. Anal. Appl. 310 (2005), no. 2, 626-640.

[9] _ Generalized Convexity and Vector Optimization, Springer-Verlag, Berlin Heidelberg, 2009.

[10] M. A. Noor, Nonconvex functions and variational inequalities, J. Optim. Theory Appl. 87 (1995), no. 3, 615-630.

[11] V. Preda, Optimality and duality in fractional multiple objective programming involving semilocally preinvex and related functions, J. Math. Anal. Appl. 288 (2003), no. 2, 365-382.

[12] V. Preda and I. M. Stancu-Minasian, Duality in multiple objective programming involving semilocally preinvex and related functions, Glas. Mat. Ser. III 32(52) (1997), no. 1, $153-165$.

[13] V. Preda, I. M. Stancu-Minasian, and A. Batatorescu, Optimality and duality in nonlinear programming involving semilocally preinvex and related functions, J. Inform. Optim. Sci. 17 (1996), no. 3, 585-596.

[14] N. G. Rueda and M. A. Hanson, Optimality criteria in mathematical programming involving generalized invexity, J. Math. Anal. Appl. 130 (1988), no. 2, 375-385.

[15] T. Weir and B. Mond, Pre-invex functions in multiple objective optimization, J. Math. Anal. Appl. 136 (1988), no. 1, 29-38.

[16] X. M. Yang and D. Li, On properties of preinvex functions, J. Math. Anal. Appl. 256 (2001), no. 1, 229-241.

[17] _ Semistrictly preinvex functions, J. Math. Anal. Appl. 258 (2001), no. 1, 287308

MONIKA JAISWAL

DST-Centre For Interdisciplinary Mathematical Sciences

Department of Mathematics

BANARAS HINDU UNIVERSITY

VARANASI-221005, INDIA

E-mail address: monikajsmj@gmail.com

Shashi Kant Mishra

DST-CENTRE FOR INTERDISCIPLINARY Mathematical SCIENCES

Department of Mathematics

BANARAS HiNdU UNIVERSITY

VARANASI-221005, INDIA

E-mail address: bhu.skmishra@gmail.com

Bader Al Shamary

Department of Mathematics

KUWAIT UNIVERSITY

SAFAT-13060, KUWAIT

E-mail address: bader231ku@yahoo.com 